\title{
An Acute Gout Attack Folllowing Parathyroidectomy
}

-Mustafa Unal ${ }^{1}$, Dilek Arpaci ${ }^{1}$, Basak Erol ${ }^{2}$, Taner Bayraktaroglu ${ }^{1}$

-1 Department of Endocrinology and Metabolism, Bulent Ecevit University Faculty of Medicine, Zonguldak, Turkey

-2Department of Internal Medicine, Bulent Ecevit University Faculty of Medicine, Zonguldak, Turkey

\section{Background}

-Gout is an arthritis characterized by the deposition of monosodium urate cyristales in the joints because of chronic hyperuricemia. Infections, intravenous contrast materials, acidosis, trauma, surgical interventions can be counted as common causes of acute gout attack. The aim of this study was to present postoperative gout attack at a patient with

parathyroidectomy •

\section{Case Report}

-65 year old man presented with back pain and fatique was admitted to our endocrinology clinic with hypercalcemia $(12.4 \mathrm{mg} / \mathrm{dl})$ for further investigation. He had a previous history of gout disease 5 years before admission but he said never treated for gout. In the laboratory examination, her urine was 136, creatinin was 3.5 , albumine was 4.1 , calcium was $12.4 \mathrm{mg} / \mathrm{dl}$, fosfor was $3 \mathrm{mg} / \mathrm{dl}$, parathormone was $1386 \mathrm{ng} / \mathrm{ml}$. In 24-hour-urine, calcium was measured as $365 \mathrm{mg}$ /day. In his neck ultrasound, it was determined a $12 * 16^{*} 14 \mathrm{~mm}$ hypoechoic lesion consistent with a parathyroid adenoma on right inferior posterior at the inferior of gland. In urinary ultrasound, there was multiple stones. Patient transferred to generel surgery department for parathyroidectomy operation. After right inferior parathyroidectomy operation,in the second postoperative day, patient had high fever raised to $39^{\circ} \mathrm{C}$, pain, swelling, limitation in movements and redness in metatarsopharyngeal joint at right foot. Other physical examinations were normal. The uric acid level was studied and measured as $11.5 \mathrm{mg} / \mathrm{dl}$. In the diagnosis, a gout attack was thought and colchium $0.5 \mathrm{mg} 3 * 1$ was began for treatment. The response to that therapy was fast. About in 24 hours fever was lower, pain was decreased and the general situation of patient gone well

\section{Conclusion}

The likely reasons for postoperative flares include fluid shifts that alter the serum urate level and may favor new medications that may elicit increases or decreases in serum urate. Gantes et al. From Spain presented two cases with pseudo-gout cases after parathyroidectomy. In their cases, gout symptoms began at second post-operative day (like our cases) and they reported that acute attack symptoms regressed with calcium replacement, using nsaid and $1 \mathrm{mg}$ /day colchium.

In peri-operative term, the gout attack can begin with many factors. Catobolic process in operative term, starvation or dehydration can induce the hyperuricemia and gout. Postoperative gout appears in generally 8 day after surgery. The affected joint part is same with the affected joint before surgery. If patients have previous gout history prepare for a surgery, examination of uric acid levels should be done and prophylactic colcium treatment should be given if needed. 